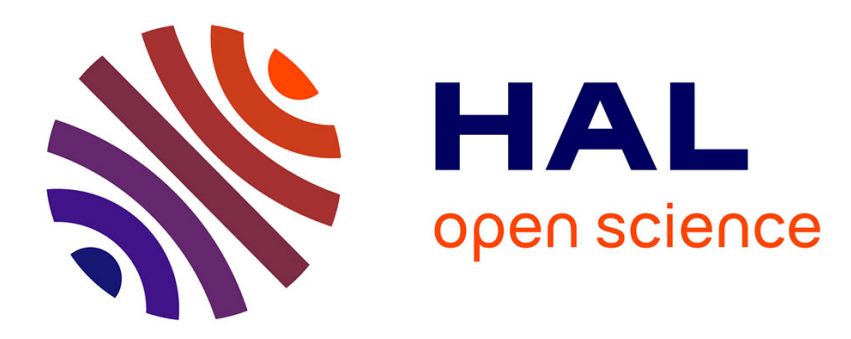

\title{
Les moustiques vecteurs d'arbovirus : une histoire sans fin
}

\author{
Anna-Bella Failloux
}

\section{To cite this version:}

Anna-Bella Failloux. Les moustiques vecteurs d'arbovirus : une histoire sans fin. Biologie Aujourd'hui, 2018, 212 (3-4), pp.89-99. 10.1051/jbio/2018026 . pasteur-03335533

\section{HAL Id: pasteur-03335533}

\section{https://hal-pasteur.archives-ouvertes.fr/pasteur-03335533}

Submitted on 6 Sep 2021

HAL is a multi-disciplinary open access archive for the deposit and dissemination of scientific research documents, whether they are published or not. The documents may come from teaching and research institutions in France or abroad, or from public or private research centers.
L'archive ouverte pluridisciplinaire HAL, est destinée au dépôt et à la diffusion de documents scientifiques de niveau recherche, publiés ou non, émanant des établissements d'enseignement et de recherche français ou étrangers, des laboratoires publics ou privés. 


\title{
Les moustiques vecteurs d'arbovirus: une histoire sans fin
}

\author{
Anna-Bella Failloux ${ }^{*}$ \\ Institut Pasteur, Département de Virologie, Arbovirus et Insectes Vecteurs, 25 rue du Dr Roux, 75724 Paris Cedex15, France
}

Reçu le 10 octobre 2019

\begin{abstract}
Résumé-L'émergence ou la ré-émergence récente des maladies à transmission vectorielle et plus précisément, celle associée aux arbovirus tels que la dengue, le chikungunya, le Zika ou encore la fièvre jaune ne sont pas des phénomènes nouveaux. Aujourd'hui, la mondialisation des échanges commerciaux, des déplacements des voyageurs ainsi que l'urbanisation anarchique de nombreuses villes tropicales et subtropicales créent les conditions propices à l'installation durable des moustiques vecteurs et par conséquent, à l'introduction des arbovirus. Cette revue décrit les principaux arbovirus importants en santé humaine et leurs vecteurs épidémiques, ainsi que les conditions facilitant leur émergence.
\end{abstract}

Mots clés : maladies à transmission vectorielle, arbovirus, moustiques, émergence, changements globaux

\begin{abstract}
Mosquitoes as vectors of arboviruses: an endless story. The recent emergence or reemergence of vector-borne diseases (VBD) and, more specifically, VBD associated with arboviruses such as dengue, chikungunya, Zika or yellow fever are not new events. The globalization of trade and travels as well as the unplanned urbanization of many tropical and subtropical cities have created the conditions suitable for the establishment of vector mosquitoes offering opportunities for arbovirus introduction. This review describes the major arboviruses important for human health and their epidemic vectors, and the conditions leading to their emergence.
\end{abstract}

Keywords: vector-borne diseases, arbovirus, mosquitoes, emergence, global changes

\section{Les facteurs favorisant la dissémination des vecteurs et de leurs agents pathogènes}

Pendant une grande partie de l'histoire de l'humanité, les populations humaines restèrent relativement isolées les unes des autres. Les premiers mouvements de populations de grande ampleur ont eu lieu lors de la traite des esclaves vers le nouveau monde introduisant son cortège d'agents pathogènes qui ont décimé les peuples autochtones (Diamond, 1997). Les guerres régionales et mondiales ont, par la suite, amorcé la mondialisation des agents pathogènes. Plus tard, l'avènement des moyens rapides de transports et, plus particulièrement, le développement de l'aviation, ont accentué la mobilité de l'homme, et ceci au même rythme que la croissance économique mondiale. Depuis 1960, le nombre de passagers a augmenté de $9 \%$ chaque année et le transport de fret a suivi la même tendance (Upham et al., 2003). De même, la mondialisation de l'économie a exacerbé les échanges maritimes de plus de $27 \%$ depuis 1993 (Zachcial \& Heideloff, 2003).

\footnotetext{
*Auteur correspondant: anna-bella.failloux@pasteur.fr
}

Ainsi, la mondialisation des échanges de personnes et de marchandises va exposer une grande partie de l'humanité aux agents pathogènes nouveaux et/ou anciens et également favoriser l'expansion rapide des insectes vecteurs qui les transmettent (Guimera et al., 2005; Perrings et al., 2005). Aujourd'hui, plus de $25 \%$ des décès annuels dans le monde sont directement liés aux maladies infectieuses et $23 \%$ de ces maladies infectieuses sont des maladies à transmission vectorielle (Jones et al., 2008). Au travers d'une cartographie des échanges commerciaux et des personnes, il est dorénavant possible d'élaborer une carte des itinéraires à haut risque d'invasion des insectes vecteurs dans le réseau de transport mondial. Ces efforts ont permis une planification des mesures de contrôle prônant des campagnes de désinsectisation des avions et autres moyens de transports (e.g. conteneurs sur bateaux) (Tatem et al., 2006).

Bien que la distance ne soit plus un facteur limitant au trafic des vecteurs, le climat constitue toujours un facteur contraignant l'installation des insectes. En effet, les insectes sont des organismes à sang froid dit poïkilothermes. Incapables de réguler leur température interne, 

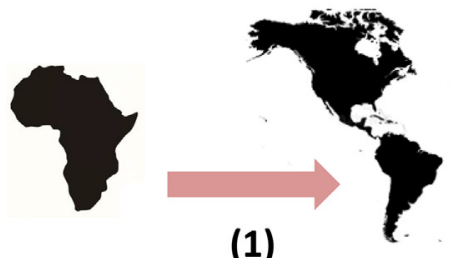

(1)

Epidémies urbaines à la suite de l'introduction d'un arbovirus

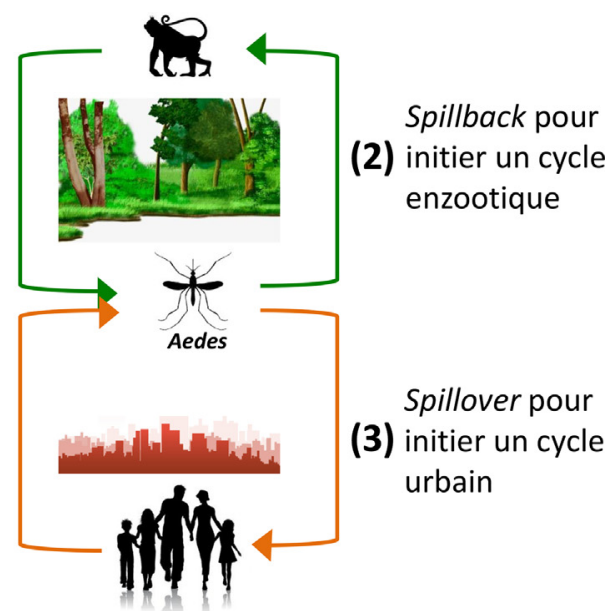

Figure 1. Les différentes étapes dans l'émergence d'un arbovirus. (1) Introduction d'un nouvel arbovirus dans une région où la population humaine est immunologiquement naïve, initiant des épidémies urbaines. (2) Spillback avec retour du virus dans un environnement «sauvage» où il circule entre des animaux sauvages (dont les primates non humains) et des moustiques zoophiles; l'homme est généralement exclu de ce cycle. (3) Spillover avec sortie du virus du cycle sauvage pour initier un cycle urbain entretenu par des moustiques anthropophiles et dont l'hôte vertébré est principalement l'homme.

les insectes s'adaptent aux changements de température en se déplaçant vers des zones où les conditions climatiques sont compatibles avec leur développement. À titre d'exemple, la distribution géographique actuelle du moustique tigre Aedes albopictus englobe des régions du monde qui présentent une similarité climatique avec Chiba au Japon, le berceau d'origine de ce moustique (Tatem et al., 2006). De plus, la durée du cycle de développement du moustique dépend de la température : à $30^{\circ} \mathrm{C}$, cette durée est deux fois plus courte qu'à $20^{\circ} \mathrm{C}$ (Delatte et al., 2009). Le taux de survie des adultes dépend également de la température (Brady et al., 2013); ce paramètre est extrêmement important car le moustique adulte infecté doit survivre suffisamment longtemps pour assurer la transmission de l'agent pathogène.

Le niveau socio-économique des pays apporte également son lot de facteurs aggravants ; par exemple, le nonaccès à l'eau potable et la mauvaise gestion des eaux usées créent des points d'eau propices au développement et à la pullulation des moustiques (Woolhouse \& GowtageSequeria, 2005). De plus, la déforestation, certaines pratiques agricoles, l'utilisation irraisonnée des insecticides sont d'autres facteurs qui favorisent la transmission des agents pathogènes par les vecteurs.

\section{L'émergence des arbovirus}

Les arbovirus (pour Arthropod-Borne Virus) sont des virus très différents d'un point de vue taxonomique mais ont en commun un cycle de transmission faisant intervenir deux hôtes, un vertébré et un arthropode. La transmission biologique ou horizontale (par opposition à la transmission verticale qui est le passage du virus de la femelle infectée à sa descendance) décrit le passage du virus d'un vecteur à un hôte vertébré après un repas de sang infectieux. Alors que la transmission verticale est un phénomène généra- lement de faible importance, la transmission horizontale est le mécanisme qui assure la circulation active du virus en situation épidémique.

La plupart des arbovirus circulent originellement au sein d'un cycle enzootique dans un environnement forestier entre des primates non humains jouant le rôle de réservoir et des populations de moustiques zoophiles. L'émergence coïncide avec la capture d'un virus selvatique par un moustique anthropo-zoophile, qui joue le rôle de vecteur relais en assurant la transmission du virus de l'animal à l'homme. Le virus peut, par la suite, circuler au sein d'un cycle épidémique/urbain entre l'homme en tant qu'hôte réservoir et amplificateur, et les moustiques anthropophiles comme vecteurs.

L'émergence d'un arbovirus pourrait se décrire selon le scénario suivant (Figure 1) : (i) introduction d'un nouvel arbovirus dans un environnement où la population humaine est réceptive, immunologiquement naïve, provoquant ainsi des épidémies urbaines (e.g., chikungunya en 2013 et Zika en 2014 en Amérique), (ii) retour du virus en milieu forestier (spillback) initiant un cycle enzootique (e.g. fièvre jaune en Amérique), et (iii) sortie du virus du cycle enzootique (spillover) initiant un cycle urbain (e.g. fièvre jaune au Brésil). Chacune de ces trois étapes fait intervenir différentes espèces de moustiques ayant une biologie et une écologie très contrastées. Néanmoins, les vecteurs épidémiques urbains sont principalement Aedes aegypti et Ae. albopictus.

\section{Les vecteurs épidémiques des arbovirus importants en santé humaine}

Les épidémies liées aux arbovirus tels que les virus de la dengue, du chikungunya, du Zika et de la fièvre jaune frappent des régions où sont présents Ae. aegypti ou Ae. albopictus (Kraemer et al., 2017). Ces deux moustiques ont 


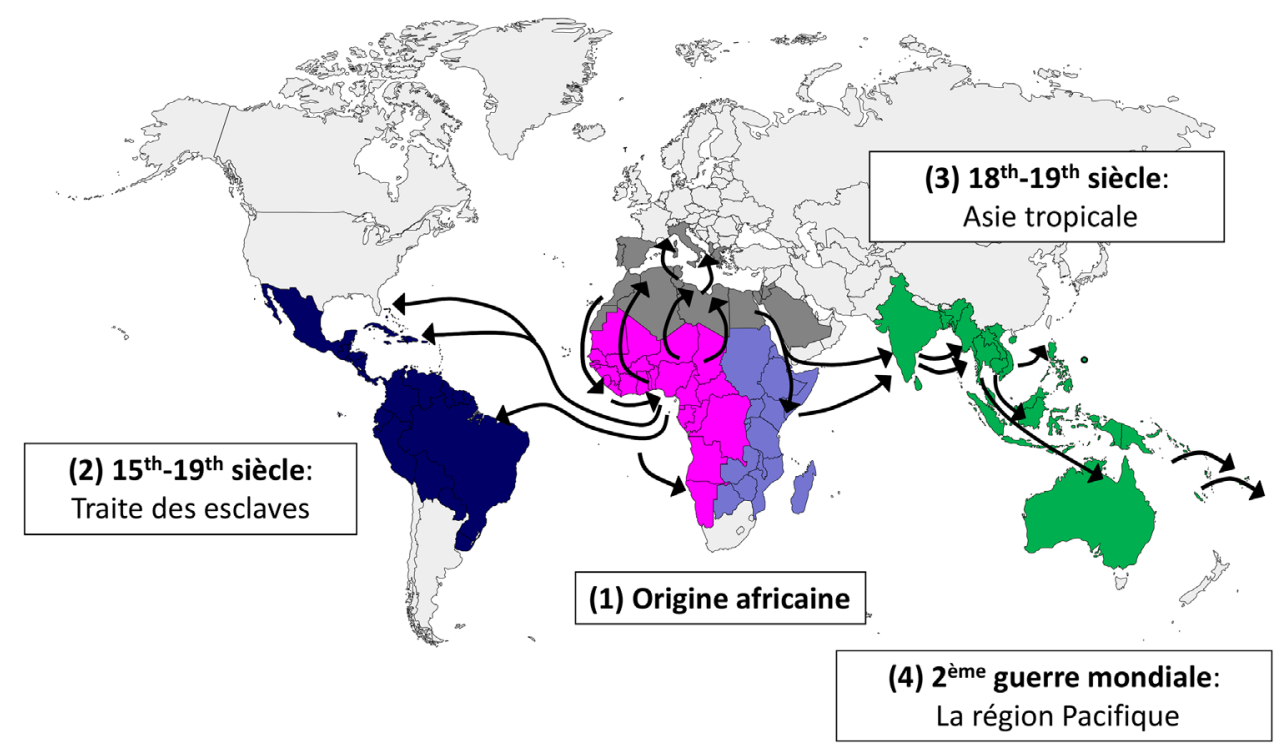

Figure 2. Histoire de la migration du moustique Aedes aegypti à partir du continent africain d'où l'espèce est originaire. (1) La forme ancestrale est présente sous la forme Aedes aegypti formosus dans les forêts tropicales d'Afrique subsaharienne. L'assèchement progressif du Sahara a contraint les populations humaines à fuir les régions arides, emportant avec eux la forme domestique (Aedes aegypti aegypti) vers l'Afrique du Nord, initiant une colonisation des pays du pourtour méditerranéen par ce moustique. (2) Â partir du $15^{\mathrm{e}}$ siècle, la traite des esclaves a introduit le moustique domestique dans les Amériques et la Caraïbe. (3) Avec l'ouverture du canal de Suez, Ae. aegypti aegypti a été introduit en Asie. (4) Le moustique a fini par coloniser les îles du Pacifique, invasion parachevée après la seconde guerre mondiale.

su exploiter l'environnement humain en prenant des repas sanguins sur l'homme et en se développant dans des gîtes larvaires créés par l'homme.

Aedes aegypti (Linneaus, 1762) est un moustique d'origine africaine (Failloux et al., 2002; Powell et al., 2018). Il se caractérise morphologiquement par la présence d'une lyre de couleur argentée et de deux lignes centrales sur le thorax. En Afrique tropicale, ce moustique est présent sous sa forme forestière, Ae. aegypti formosus, de coloration sombre colonisant principalement des gites larvaires d'origine naturelle (creux d'arbres, trous de rochers..). Hors d'Afrique, l'espèce est présente sous forme de populations domestiques dans la région intertropicale: Ae. ae. aegypti y est hautement anthropophile colonisant des gîtes artificiels tels les pots de fleurs, les pneus, les gouttières...

L'histoire d'Ae. aegypti suit celle de l'homme. L'assèchement du Sahara a contraint les populations humaines à fuir les zones arides et à stocker l'eau dans des contenants artificiels lors de leurs déplacements. Ces contenants sont devenus ainsi des sites privilégiés de prolifération des moustiques favorisant la différenciation de moustiques plus adaptés à un environnement «humain ». À l'occasion des vagues successives de migrations humaines, la forme domestique Ae. aegypti aegypti a envahi la ceinture intertropicale et même au-delà : (i) le nouveau monde lors de la traite des esclaves dès le $15^{\mathrm{e}}$ siècle, (ii) le bassin méditerranéen où l'espèce était encore présente dans les ports européens jusqu'en 1950, (iii) l'Asie depuis le bassin méditerranéen dès l'ouverture du canal de Suez en 1869, (iv) et la région du Pacifique Sud dès 1904 (Powell et al.,
2018) (Figure 2). Aujourd'hui, le transport d'Ae. ae. aegypti continue sans sens privilégié grâce à l'intensification des échanges commerciaux.

En raison de leur forte domestication, les femelles d' $A e$. aegypti pondent leurs œufs dans des gîtes artificiels, tels que des réservoirs d'eau, des pots de fleurs et des pneus usagés. Il a été démontré que les œufs pouvaient résister à la dessiccation pendant au moins un an (Rezende et al., 2008). Les formes pré-imaginales d'Ae. aegypti s'adaptent aussi à des conditions climatiques extrêmes: les larves peuvent rester viables à très basses températures et certaines populations se maintiennent à des températures de plus de $40^{\circ} \mathrm{C}$.

Aedes albopictus (Skuse, 1894) ou «moustique tigre», est originaire d'Asie du Sud-Est. Ce moustique, fréquent en Asie, a été décrit pour la première fois à Calcutta en Inde. Avant 1979, Ae. albopictus s'étendait du Pacifique à l'Océan Indien (Madagascar) et il est fort probable qu'Ae. albopictus ait accompagné les vagues successives de colonisation du Sud-Ouest de l'Océan Indien par les peuples d'origine indonésienne (Raharimalala et al., 2012). On le retrouve également en Chine, au Japon et en Corée. L'Europe est touchée à partir de 1979, date à laquelle on le trouve pour la première fois en Albanie, certainement à la suite d'une importation via le commerce de pneus en provenance de Chine (Adhami \& Reiter, 1998). En 1990, le moustique est retrouvé à Gênes où il réussit à s'implanter et devient une cause de nuisance en Italie (Dalla Pozza \& Majori, 1992). L'espèce est actuellement présente dans 20 pays européens (Medlock et al., 2012) et notamment, dans 51 départements en 

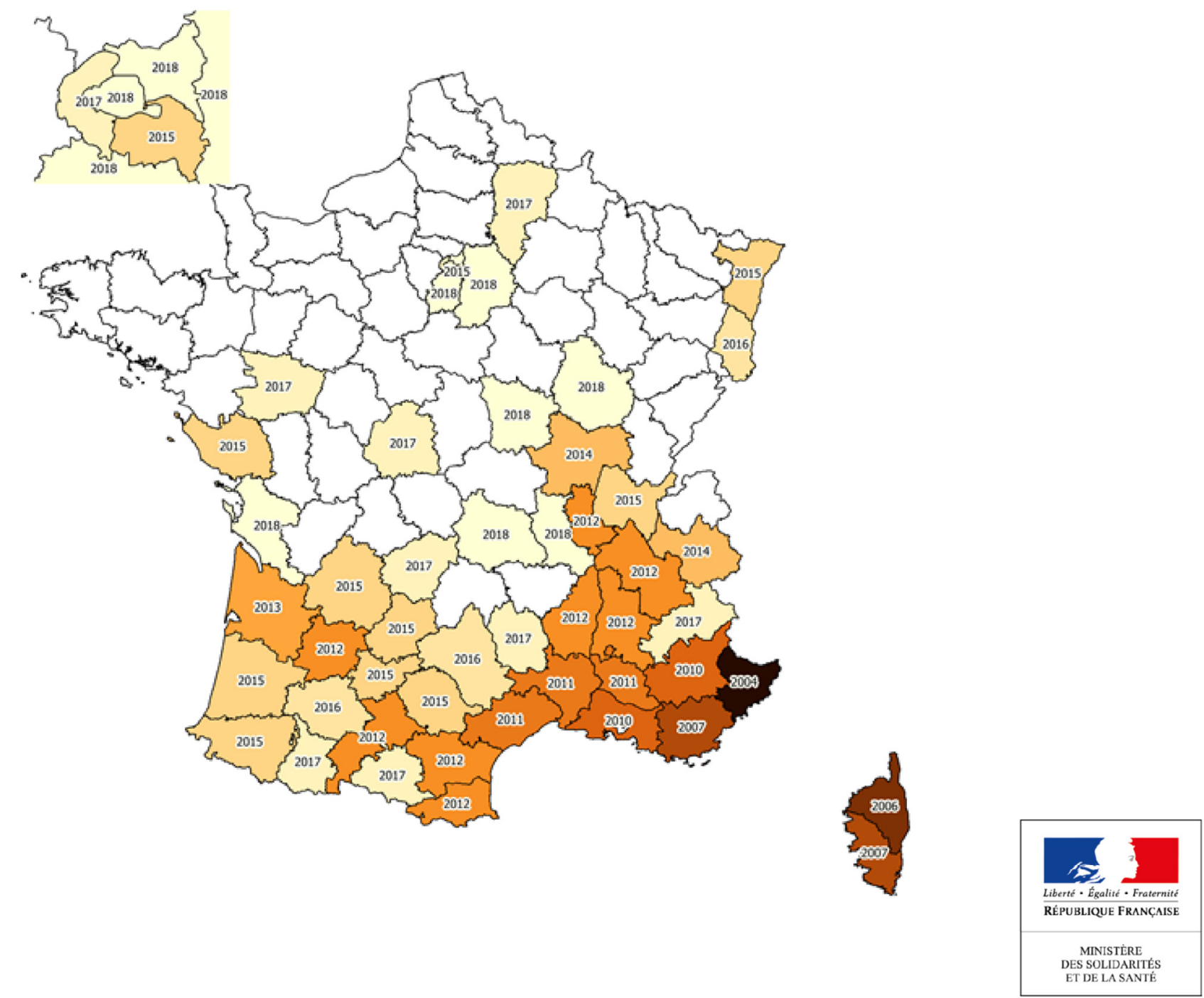

Figure 3. Carte de France indiquant l'aire de distribution d'Aedes albopictus. La date où chaque département a été colonisé par l'espèce est indiquée. Source: Ministère des Solidarités et de la Santé.

France (https://solidarites-sante.gouv.fr/sante-et-envi ronnement/risques-microbiologiques-physiques-et-chimi ques/especes-nuisibles-et-parasites/article/cartes-de-pre sence-du-moustique-tigre-aedes-albopictus-en-francemetropolitaine). Bien que l'espèce ait été identifiée pour la première fois en France en Normandie en 1999 (Schaffner \& Karch, 2000), ce n'est que depuis 2004 qu' Ae. albopictus s'est implanté durablement dans le sud-est de la France (Delaunay et al., 2009) (Figure 3).

Ae. albopictus a fini par s'installer aux États-Unis en 1985 (Hawley et al., 1987) puis s'est très vite propagé à travers le pays jusqu'au Mexique. Il a également envahi l'Amérique du Sud et notamment, le Brésil à partir de 1986 (Forattini, 1986). Ae. albopictus a été détecté dans certains pays d'Afrique tels le Nigéria (Savage et al., 1992), le Cameroun (Fontenille \& Toto, 2001), le Gabon (Coffinet et al., 2007), l'Afrique du Sud (Cornel \& Hunt,1991), la Guinée Équatoriale (Toto et al., 2003). L'aptitude de ce moustique à coloniser de nouvelles aires géographiques et à s'y installer traduit sa grande plasticité écologique. Sa capacité à s'implanter dans des zones tempérées représente une crainte quant à l'introduction des arboviroses que l'espèce peut transmettre. En effet, Ae. albopictus est capable de survivre aux basses températures de l'hiver sous forme d'œufs en diapause qui écloront lors des pluies printanières (Hawley et al., 1987 ; Hanson \& Craig, 1994).

Les deux espèces, Ae. aegypti et Ae. albopictus, sont amenées très souvent à partager la même niche écologique, par exemple en Amérique du Nord, en Amérique du Sud, en Afrique Centrale et dans la région de l'Océan Indien. Dans certains cas, Ae. albopictus présente une aptitude compétitive supérieure qui le favorise vis-à-vis d'Ae. aegypti (Paupy et al., 2009).

\section{Les moustiques ne sont pas tous vecteurs}

On compte au moins 3500 espèces de moustiques et tous ne sont pas vecteurs. Pour transmettre un arbovirus, le moustique doit être génétiquement compétent. La 


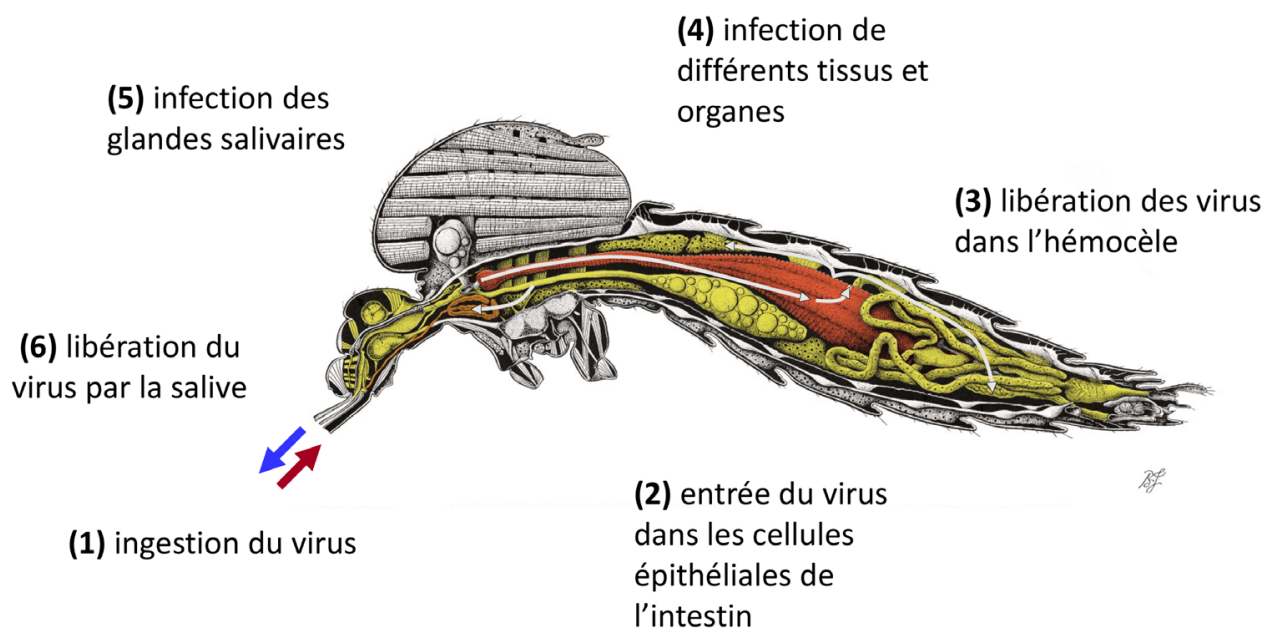

Figure 4. Différentes étapes d'infection du moustique par un arbovirus. Après ingestion du repas sanguin (1), le virus pénètre dans la cellule épithéliale et se multiplie (2). Les virus néoformés sont alors libérés dans la cavité générale du moustique (3) où il infecte différents organes (4). Une fois que les glandes salivaires sont infectées (5), le virus peut être émis avec la salive libérée par le moustique lors d'une piqûre (6).

compétence vectorielle est la capacité d'un insecte à ingérer le virus au cours d'un repas de sang, à assurer la réplication virale et à transmettre le virus à un hôte vertébré approprié (Kramer \& Ebel, 2003). De façon plus détaillée, le cheminement du virus dans le moustique vecteur comporte plusieurs étapes: (i) ingestion du virus par la femelle lors d'un repas sanguin sur un hôte vertébré en phase de virémie, (ii) entrée du virus dans les cellules épithéliales de l'intestin grâce à un récepteur membranaire, (iii) après réplication, libération des virions néoformés dans l'hémocèle, cavité générale où les organes internes du moustique baignent dans l'hémolymphe, (iv) infection de différents tissus et organes (le tissu nerveux, le corps gras, les organes reproducteurs), (v) infection des glandes salivaires, et (vi) libération du virus par la salive émise par la femelle lors de la piqûre (Figure 4). Le temps qui s'écoule entre l'ingestion du repas infectieux et le moment où le moustique devient apte à transmettre, les particules virales étant présentes dans la salive, correspond à la période d'incubation extrinsèque (PIE) (Ruckert \& Ebel, 2018). Ce paramètre est extrêmement important car le moustique doit survivre au-delà de la durée de la PIE pour pouvoir transmettre le virus. De plus, la durée de la PIE dépend de la température: lorsqu'on augmente la température, on diminue la durée de la PIE (Rohani et al., 2009). En d'autres termes, lorsque la température augmente, la transmission aura tendance à augmenter.

L'évaluation de la compétence des vecteurs débute par la récolte de moustiques sur le terrain sous forme d'œufs, larves et nymphes et leur élevage pour obtenir des adultes. Les femelles ainsi obtenues sont infectées grâce à un système utilisant une membrane artificielle couvrant la base d'une capsule contenant le sang infecté maintenu à $37^{\circ} \mathrm{C}$ (système Hemotek ${ }^{\circledR}$ ). Au terme du repas, seules les femelles complètement gorgées sont incubées jusqu'à leur analyse. Pour définir la compétence du vecteur, trois paramètres sont mesurés: (i) le taux d'infection corres- pondant à la proportion de moustiques présentant un intestin moyen infecté, (ii) le taux de dissémination se référant à la proportion de femelles capables d'assurer la dissémination virale au-delà de l'intestin moyen (ceci est possible en détectant le virus dans la tête, les pattes ou les ailes des moustiques), et (iii) le taux de transmission représentant la proportion de moustiques portant le virus détecté dans la salive.

La capacité vectorielle, quant à elle, est une mesure de l'efficacité du vecteur à transmettre un agent infectieux en conditions naturelles (Kramer \& Ebel, 2003). Elle dépend de facteurs intrinsèques et extrinsèques qui agissent sur la dynamique du cycle de transmission virus-vecteur-hôte vertébré. Parmi les paramètres intrinsèques, on peut citer la compétence vectorielle et la longévité des femelles, de même que leurs préférences trophiques et la fréquence des repas sanguins. Les facteurs extrinsèques regroupent la disponibilité de l'hôte vertébré et son état immunitaire, ainsi que l'abondance de vecteurs. Cette dernière est liée à la température et à la pluviosité ou en d'autres termes, à la saison.

\section{Les principales arboviroses liées aux moustiques du genre Aedes \\ La dengue (Figure 5A)}

La dengue est l'arbovirose la plus importante en termes de morbidité et mortalité avec 390 millions de cas chaque année dont 96 millions développant des signes cliniques sévères (Bhatt et al., 2013). Le virus de la dengue (DENV) appartient au genre Flavivirus de la famille des Flaviviridae; il possède un génome formé d'un simple brin ARN de polarité positive d'une longueur de $11 \mathrm{~kb}$. Quatre sérotypes (DENV-1 à DENV-4) sont principalement décrits, se subdivisant chacun en plusieurs génotypes. L'immunité acquise à la suite de l'infection par l'un des 
(A)

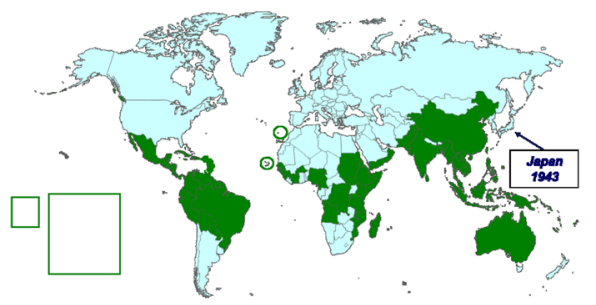

(C)

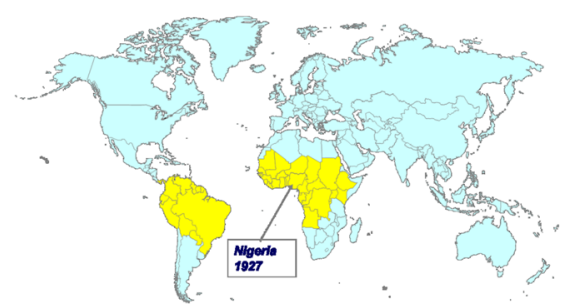

(B) Chikungunya

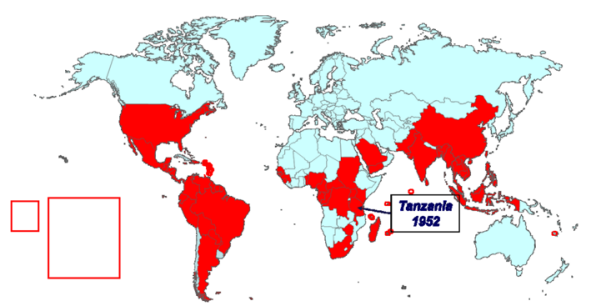

(D) Zika

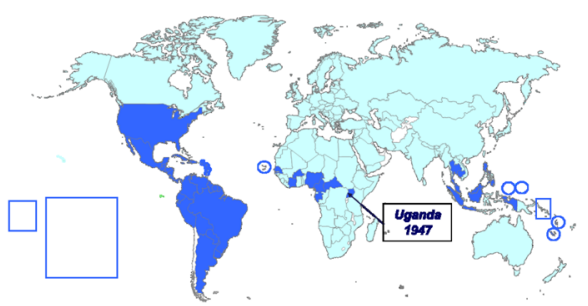

Figure 5. Les quatre arbovirus les plus importants pour la santé humaine : dengue (A), chikungunya (B), Zika (C) et fièvre jaune (D). Sont indiqués les aires de distribution et le lieu/date du premier isolement.

sérotypes confère une immunité protectrice contre le sérotype infectant mais pas contre les autres sérotypes (Vasilakis \& Weaver, 2008).

Les tableaux cliniques liés à la dengue vont de la forme asymptomatique, la forme classique (DF pour Dengue Fever) à la dengue hémorragique (DHF pour Dengue Hemorragic Fever). Il est à noter que $80 \%$ des cas d'infections sont asymptomatiques. Demeurant infectés et non malades, ces cas participent activement à la transmission et la diffusion du virus (Duong et al., 2015; Ten Bosch et al., 2018).

La dengue sévit principalement dans la région intertropicale. Sa ré-émergence au cours des 50 dernières années est étroitement liée à l'urbanisation, l'intensification des voyages, et une lutte anti-vectorielle inadéquate (Gubler, 2011). Hors des régions traditionnellement endémiques pour la dengue, une transmission autochtone a aujourd'hui été décrite aux États-Unis (Bouri et al., 2012), en France (La Ruche et al., 2010), en Croatie (Gjenero-Margan et al., 2011) et à Madère (Tomasello \& Schlagenhauf, 2013), initiée partout par des cas importés.

$A$ e. aegypti est le vecteur principal de la dengue mais dans des régions où il est absent ou peu fréquent, c'est $A e$. albopictus qui joue alors le rôle de vecteur. Il était impliqué dans des épidémies survenues dans les années 1970, aux Seychelles et à la Réunion, et dans les années 2000, à Macao et à Hawaï (Gratz, 2004; Effler et al., 2005).

\section{Le chikungunya (Figure 5B)}

Le virus du chikungunya (CHIKV) est un autre arbovirus transmis par Ae. aegypti et Ae. albopictus. Il a été isolé pour la première fois en Tanzanie en 1952. Le CHIKV appartient au genre Alphavirus de la famille des Togaviridae. Son génome est composé d'un simple brin
d'ARN de polarité positive d'environ 11,8 kb. Trois génotypes sont décrits: Est-Centre-Sud-africain (ECSA), Ouest-africain et asiatique. Les symptômes de la maladie sont une forte fièvre, des atteintes articulaires, des maux de tête, des douleurs musculaires, une éruption cutanée. Des formes neurologiques graves sont exceptionnellement décrites.

Endémique à l'Afrique et l'Asie, ce virus a émergé en 2004, provoquant des épidémies dans les îles de la région de l'Océan Indien. Dès 2006, le génotype ECSA a été à l'origine des épidémies liées au moustique Ae. albopictus, rarement décrit jusque-là comme vecteur de CHIKV. Il a été démontré qu'un seul changement d'acide aminé au niveau de la glycoprotéine E1 de l'enveloppe virale est à l'origine de cette adaptation (Schuffenecker et al., 2006 ; Tsetsarkin et al., 2007; Vazeille et al., 2007).

En octobre 2013, les premiers cas autochtones ont été recensés dans la Caraïbe sur l'île de Saint-Martin. Ae. aegypti est le seul vecteur présent assurant la transmission d'un CHIKV de génotype asiatique. Depuis, près de 46 pays d'Amérique ont été touchés avec plus de 3 millions de personnes atteintes (Yactayo et al., 2016). De façon exceptionnelle, les premiers cas autochtones ont été recensés en Europe : en Italie en 2007, 2017 et en France en 2010, 2014, 2017 (Angelini et al., 2007; Tomasello \& Schlagenhauf, 2013; Venturi et al., 2017) (http://invs. santepubliquefrance.fr/fr../Dossiers-thematiques/Mala dies-infectieuses/Maladies-a-transmission-vectorielle/ Chikungunya/Donnees-epidemiologiques).

\section{La fièvre jaune (Figure $5 \mathrm{C}$ )}

Le virus de la fièvre jaune (FJV) est un prototype du genre Flavivirus de la famille des Flaviviridae. Le virus a été isolé pour la première fois en Afrique de l'Ouest en 
1927. Son génome est composé d'une molécule d'ARN simple brin de polarité positive d'environ $11 \mathrm{~kb}$. À ce jour, on a décrit 7 génotypes de FJV se différenciant par au plus $9 \%$ de nucléotides. En Afrique, on a répertorié 5 génotypes et seulement deux en Amérique. Les souches d'Amérique du Sud sont phylogénétiquement très proches de celles d'Afrique de l'Ouest suggérant une origine africaine des souches américaines, certainement introduites lors de la traite des esclaves (Bryant et al., 2007).

Les symptômes (fièvre, douleurs musculaires, maux de tête) peuvent alors évoluer vers des formes graves avec survenue d'un syndrome hémorragique. La mort survient dans 50 à $80 \%$ des cas. Chaque année, environ 200000 cas humains sont recensés dont $90 \%$ en Afrique. Cette région regroupe 34 pays africains et 500 millions d'habitants exposés. Depuis 20 ans, au moins une épidémie est rapportée chaque année dans cette région malgré la disponibilité d'un vaccin efficace, le vaccin 17D.

Sur le continent américain, les premières épidémies de fièvre jaune ont été observées au $17^{\mathrm{e}}$ siècle. Au début du $20^{\mathrm{e}}$ siècle, la détermination du rôle d'Ae. aegypti comme vecteur par le médecin cubain Carlos Finlay, confirmé par la suite par le médecin américain Walter Reed, a permis d'entreprendre le contrôle de la fièvre jaune en éradiquant le moustique. La fondation Rockefeller puis la Pan American Health Organisation (PAHO) parvinrent à contrôler $A e$. aegypti dans une grande partie de l'Amérique tropicale. Le cycle urbain de la fièvre jaune n'est plus décrit en Amérique tropicale à partir de 1942. Dans les forêts de l'Amazonie, il n'existe aujourd'hui qu'un cycle selvatique où le virus circule entre des singes américains pouvant mourir de la fièvre jaune et des moustiques zoophiles. L'homme peut occasionnellement contracter le virus par contact avec les vecteurs selvatiques du genre Haemagogus. Depuis les années 1970 où le contrôle d'Ae. aegypti a été interrompu, une ré-introduction massive de cette espèce crée de nouveau une situation très préoccupante qui fait craindre le retour des épidémies de fièvre jaune urbaines en Amérique du Sud. Par ailleurs, Ae. albopictus est arrivé en Amérique récemment: aux États-Unis en 1985 et au Brésil, en 1986. Jusqu'à présent, il n'a pas été prouvé qu'Ae. albopictus pouvait participer à une épidémie de fièvre jaune en Amérique du Sud et notamment, au Brésil mais on sait qu'il est expérimentalement capable de transmettre le FJV (Couto-Lima et al., 2017; Amraoui et al., 2018). Il pourrait jouer un rôle dans le processus d'épidémisation urbaine de la fièvre jaune en servant d'intermédiaire entre le cycle selvatique et un potentiel cycle urbain.

En Afrique de l'Ouest, Ae. aegypti a été impliqué dans la transmission de la fièvre jaune urbaine alors qu'il ne l'est pas en Afrique de l'Est et du Centre où des moustiques du complexe Aedes simpsoni sont plutôt incriminés. Le principal vecteur forestier en Afrique est Aedes africanus qui assure la transmission de la fièvre jaune à l'homme.

\section{La dernière pandémie : le Zika (Figure 5D)}

Le virus Zika (ZIKV) a été isolé en 1947 de la forêt Zika en Ouganda d'un singe rhésus durant une campagne de surveillance des vecteurs de la fièvre jaune. Il a été également isolé de moustiques zoophiles, Ae. africanus. Les premiers cas humains ont été signalés seulement cinq ans plus tard, en 1952 (Fauci \& Morens, 2016). ZIKV est un Flavivirus de la famille des Flaviviridés comme la dengue et la fièvre jaune. Le génome est une molécule d'ARN simple brin de polarité positive d'environ 11$12 \mathrm{~kb}$. Il existe trois génotypes: Est-africain, Ouestafricain et asiatique.

Il est décrit dans les régions tropicales d'Asie du SudEst et d'Afrique. Les symptômes sont une fièvre, des éruptions maculo-papuleuses, des myalgies et arthralgies. Toutes les manifestations disparaissent en une semaine au maximum. Des cas de syndrome de Guillain-Barré et de microcéphalies des nouveau-nés ont été rapportés lors des dernières épidémies (Rasmussen et al., 2016).

ZIKV a émergé dans le Pacifique, sur l'île de Yap (Micronésie) en 2007 (Duffy et al., 2009) puis en Polynésie française en 2013 et autres îles du Pacifique (Musso et al., 2014). En 2015, les premiers cas symptomatiques ont été décrits au Brésil et le virus s'est ensuite propagé dans une grande partie de l'Amérique latine et les Caraïbes (Faria et al., 2016). Le virus semble avoir été introduit à plusieurs reprises en 2013 ou 2014 par des voyageurs virémiques (Faria et al., 2016). Le vecteur majeur est Ae. aegypticar il satisfait à quatre critères clés : (i) forte anthropophilie (i.e. pique principalement l'homme), (ii) espèce d'écologie urbaine vivant dans l'environnement humain, (iii) détection de moustiques naturellement infectés par ZIKV (Ferreira-de-Brito et al., 2016), et (iv) transmission expérimentale par le moustique (Chouin-Carneiro et al., 2016). En évaluant la compétence vectorielle de différentes populations d'Ae. aegypti provenant de Martinique, Guadeloupe, Guyane française, États-Unis, Brésil vis-àvis du ZIKV de génotype asiatique, on note que le virus se dissémine dans la cavité générale du moustique dès le $4^{\mathrm{e}}$ jour après l'infection (Chouin-Carneiro et al., 2016). Quant à Ae. albopictus, cette espèce assure une moins bonne dissémination virale. Contre toute attente, les deux espèces présentent une faible capacité à transmettre le virus avec moins de $10 \%$ des moustiques contenant du virus dans la salive, 14 jours après l'infection (ChouinCarneiro et al., 2016) (Figure 6). Par ailleurs, la communauté scientifique s'est mobilisée pour s'interroger sur le rôle potentiel du moustique Culex quinquefasciatus fortement présent en milieux urbains (Roundy et al., 2017). L'infection par voie orale ou inoculation intrathoracique ne permet pas de démontrer la transmission du ZIKV par les moustiques Culex (pipiens et quinquefasciatus) (Amraoui et al., 2016). Par ailleurs, les Culex infectés par ZIKV ne sont pas capables de déclencher la voie ARN interférence qui est la voie antivirale majeure; aucuns petits ARN ciblant spécifiquement ZIKV n'ont été détectés chez Culex (Lourenço-de-Oliveira \& Failloux, 2017). Donc, Cx. pipiens et Cx. quinquefasciatus ne sont pas vecteurs de ZIKV.

Hormis l'Europe, ZIKV a été présent sur tous les continents. Quel pouvait être le risque pour l'Europe à la saison d'activité du moustique Ae. albopictus? La plus 

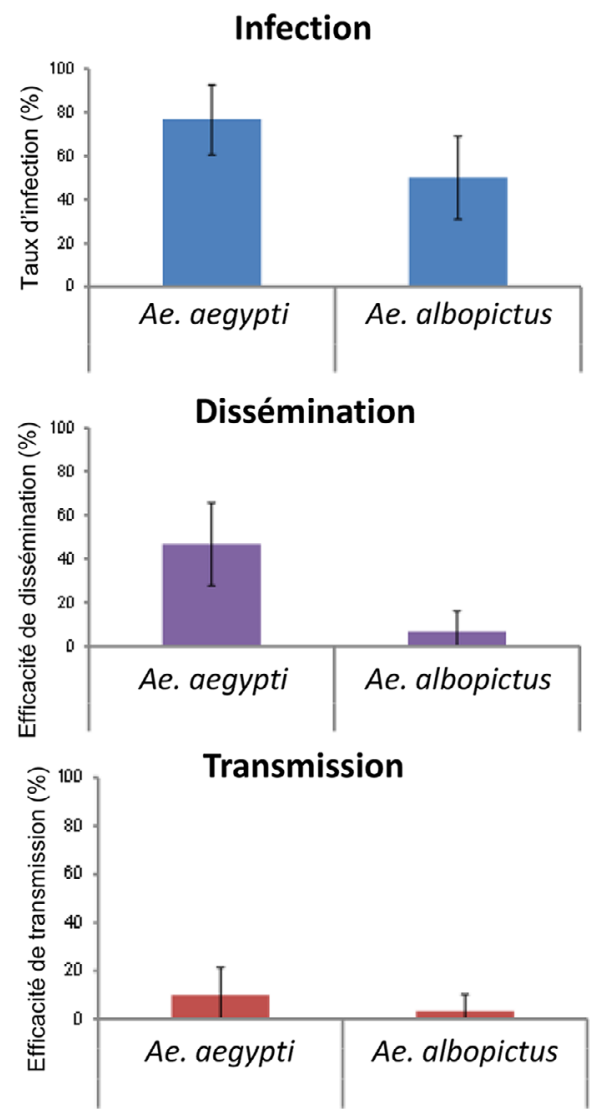

Figure 6. Infection, dissémination et transmission du virus Zika par Aedes aegypti et Aedes albopictus. Les moustiques sont examinés 14 jours après la prise d'un repas sanguin proposé à un titre de $10^{7}$ TCID50/mL. Source : Chouin-Carneiro et al. (2016).

forte probabilité d'introduction viendrait de voyageurs de retour des départements français d'Amérique où ZIKV était présent (Martinique, Guadeloupe, Guyane française). En testant la compétence vectorielle d'Ae. albopictus de France vis-à-vis du ZIKV de génotype asiatique, il a été démontré qu'Ae. albopictus pouvait disséminer le virus dès le $9^{\mathrm{e}}$ jour après infection mais ne transmettait qu'au $14^{\mathrm{e}}$ jour (Lourenço-de-Oliveira \& Failloux, 2017) (Figure 7). Ainsi, les populations d'Ae. albopictus du Sud de la France ne deviennent infectantes que deux semaines après l'infection. Pour rappel, pour $A e$. albopictus, la durée de la PIE est de $4-10$ jours avec le DENV (Xiao et al., 2014) et de 2-3 jours avec le CHIKV (Dubrulle et al., 2009). Par conséquent, Ae. albopictus de France est moins sensible au ZIKV et le risque d'une transmission autochtone de ZIKV en France et plus largement, en Europe, est relativement faible (Di Luca et al., 2016; Jupille et al., 2016).

\section{Conclusion}

Au cours de ces dernières décennies, le volume des échanges intercontinentaux de marchandises et de passagers a augmenté. Cette mondialisation a contribué à la propagation des agents pathogènes, de leurs vecteurs et de leurs hôtes, aggravant la situation des maladies à
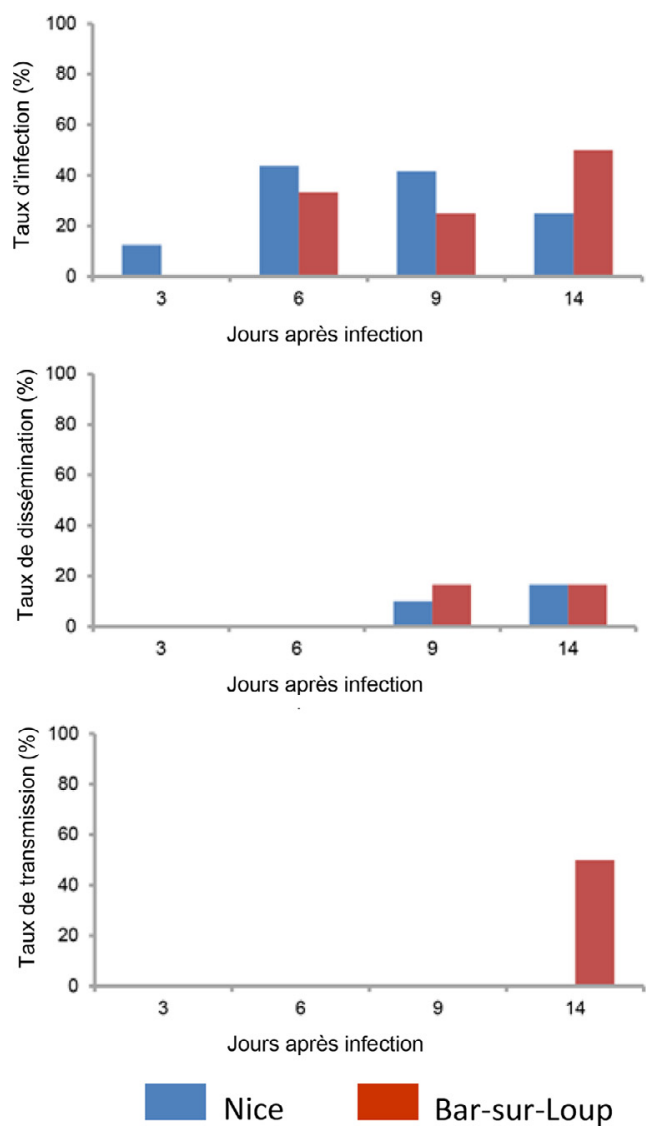

Figure 7. Compétence vectorielle d'Aedes albopictus de France pour le virus Zika. L'infection, la dissémination et la transmission du virus sont examinés 3,6 , 9 , et 14 jours après la prise d'un repas sanguin proposé à un titre de $10^{7}$ TCID $50 / \mathrm{mL}$. Source: Jupille et al. (2016).

transmission vectorielle dans les pays déjà affectés et les introduisant dans les pays occidentaux. Les effets de ces changements globaux n'en sont qu'à leurs débuts. Les villes actuelles qui concentrent plus de la moitié de l'humanité et où règnent les conditions propices au développement des moustiques vecteurs sont des bombes à retardement. En l'absence de vaccins et de traitements spécifiques, le contrôle des vecteurs reste l'ultime action à mener. Néanmoins, les mesures actuelles de lutte contre les vecteurs sont globalement inefficaces, ouvrant ainsi des champs d'étude sur des méthodes alternatives qui restent encore à être éprouvées.

Remerciements. Les travaux sur Zika ont reçu un financement de l'Union Européenne (H2020, ZIKAlliance grant agreement no. 734548$)$.

\section{Références}

Adhami, J., Reiter, P. (1998). Introduction and establishment of Aedes (Stegomyia) albopictus skuse (Diptera: Culicidae) in Albania. J Am Mosq Control Assoc, 14, 340-343.

Amraoui, F., Atyame-Nten, C., Vega-Rua, A., Lourenco-deOliveira, R., Vazeille, M., Failloux, A.B. (2016). Culex 
mosquitoes are experimentally unable to transmit Zika virus. Euro Surveill, 21(35).

Amraoui, F., Pain, A., Piorkowski, G., Vazeille, M., Couto-Lima, D., de Lamballerie, X., Lourenço-de-Oliveira, R., Failloux, A. B. (2018). Experimental adaptation of the yellow fever virus to the mosquito Aedes albopictus and potential risk of urban epidemics in Brazil, South America. Sci Rep, 8, 14337.

Angelini, R., Finarelli, A.C, Angelini, P., Po, C., Petropulacos, K., Macini, P., Fortuna, C., Venturi, G., Magurano, F., Fiorentini, C., Marchi, A., Benedetti, E., Bucci, P., Boros, S., Romi, R., Majori, G., Ciufolini, M.G., Nicoletti, L., Rezza, G., Cassone, A. (2007). An outbreak of chikungunya fever in the province of Ravenna, Italy. Euro Surveill, 12, E070906. 1.

Bhatt, S., Gething, P.W., Brady, O.J., Messina, J.P., Farlow, A. W., Moyes, C.L., Drake, J.M., Brownstein, J.S., Hoen, A.G, Sankoh, O., Myers, M.F., George, D.B., Jaenisch, T., Wint, G.R., Simmons, C.P, Scott, T.W., Farrar, J.J., Hay, S.I. (2013). The global distribution and burden of dengue. Nature, 496, 504-507.

Bouri, N., Sell, T.K., Franco, C., Adalja, A.A, Henderson, D.A., Hynes, N.A. (2012). Return of epidemic dengue in the United States: implications for the public health practitioner. Public Health Rep, 127, 259-266.

Brady, O.J., Johansson, M.A., Guerra, C.A., Bhatt, S., Golding, N., Pigott, D.M., Delatte, H., Grech, M.G., Leisnham, P.T., Maciel-de-Freitas, R., Styer, L.M., Smith, D.L., Scott, T.W., Gething, P.W., Hay, S.I. (2013). Modelling adult Aedes aegypti and Aedes albopictus survival at different temperatures in laboratory and field settings. Parasit Vectors, 6, 351.

Bryant, J.E., Holmes, E.C., Barrett, A.D. (2007). Out of Africa: a molecular perspective on the introduction of yellow fever virus into the Americas. PLoS Pathog, 3, e75.

Chouin-Carneiro, T., Vega-Rua, A., Vazeille, M., Yebakima, A., Girod, R., Goindin, D., Dupont-Rouzeyrol, M., Lourenço-deOliveira, R., Failloux, A.B. (2016). Differential susceptibilities of Aedes aegypti and Aedes albopictus from the Americas to Zika Virus. PLoS Negl Trop Dis, 10, e0004543.

Coffinet, T., Mourou, J.R., Pradines, B., Toto, J.C., Jarjaval, F., Amalvict, R., Kombila, M., Carnevale, P., Pages, F. (2007). First record of Aedes albopictus in Gabon. J Am Mosq Control Assoc, 23, 471-472.

Cornel, A.J., Hunt, R.H. (1991). Aedes albopictus in Africa? First records of live specimens in imported tires in Cape Town. J Am Mosq Control Assoc, 7, 107-108.

Couto-Lima, D., Madec, Y., Bersot, M.I., Campos, S.S., Motta, M.A., Santos, F.B.D., Vazeille, M., Vasconcelos, P.F.D.C., Lourenço-de-Oliveira, R., Failloux, A.B. (2017). Potential risk of re-emergence of urban transmission of Yellow Fever virus in Brazil facilitated by competent Aedes populations. Sci Rep, 7, 4848.

Dalla Pozza, G., Majori, G. (1992). First record of Aedes albopictus establishment in Italy. J Am Mosq Control Assoc, 8, 318-320.

Delatte, H., Gimonneau, G., Triboire, A., Fontenille, D. (2009). Influence of temperature on immature development, survival, longevity, fecundity, and gonotrophic cycles of Aedes albopictus, vector of chikungunya and dengue in the Indian Ocean. J Med Entomol, 46, 33-41.

Delaunay, P., Jeannin, C., Schaffner, F., Marty, P. (2009). [News on the presence of the tiger mosquito Aedes albopictus in metropolitan France]. Arch Pediatr, 16, Suppl 2,S66-S71.

Diamond, J. (1997). Guns, germs, and steel: the fates of human societies, in: W.W. Norton (Ed.), University of California.

Di Luca, M., Severini, F., Toma, L., Boccolini, D., Romi, R., Remoli, M.E., Sabbatucci, M., Venturi, G., Rezza, G., Fortuna, C. (2016). Experimental studies of susceptibility of Italian Aedes albopictus to Zika virus. Euro Surveill, 21(18).
Dubrulle, M., Mousson, L., Moutailler, S., Vazeille, M., Failloux, A.B. (2009). Chikungunya virus and Aedes mosquitoes: saliva is infectious as soon as two days after oral infection. PLoS One, 4, e5895.

Duffy, M.R., Chen, T.H., Hancock, W.T., Powers, A.M., Kool, J. L., Lanciotti, R.S., Pretrick, M., Marfel, M., Holzbauer, S., Dubray, C., Guillaumot, L., Griggs, A., Bel, M., Lambert, A.J., Laven, J., Kosoy, O., Panella, A., Biggerstaff, B.J., Fischer, M., Hayes, E.B. (2009). Zika virus outbreak on Yap Island, Federated States of Micronesia. N Engl J Med, 360, 2536-2543.

Duong, V., Lambrechts, L., Paul, R.E., Ly, S., Lay, R.S., Long, K. C., Huy, R., Tarantola, A., Scott, T.W., Sakuntabhai, A., Buchy, P. (2015). Asymptomatic humans transmit dengue virus to mosquitoes. Proc Natl Acad Sci USA, 112, 14688-14693.

Effler, P.V., Pang, L., Kitsutani, P., Vorndam, V., Nakata, M., Ayers, T., Elm, J., Tom, T., Reiter, P., Rigau-Perez, J.G., Hayes, J.M., Mills, K., Napier, M., Clark, G.G., Gubler, D.J., Hawaii Dengue Outbreak Investigation Team. (2005). Dengue fever, Hawaii, 2001-2002. Emerg Infect Dis, 11, 742-749.

Failloux, A.B., Vazeille, M., Rodhain, F. (2002). Geographic genetic variation in populations of the dengue virus vector Aedes aegypti. Med Vet Entomol, 16, 456-460.

Faria, N.R., Azevedo, R.D.S.D.S., Kraemer, M.U.G., Souza, R., Cunha, M.S., Hill, S.C., Thézé, J., Vasami, F.G.D.S., Macedo, F.L.L., Suzuki, A., Rodrigues, S.G., Cruz, A.C.R., Nunes, B.T., Medeiros, D.B.A., Rodrigues, D.G., Queiroz, A. L.N., da Silva, E.V.P., Henriques, D.F., da Rosa, E.S.T., de Oliveira, C.S., Martins, L.C., Vasconcelos, H.B., Casseb, L. M.N., Simith, D.B., Messina, J.P., Abade, L., Lourenço, J., Alcantara, L.C.J., de Lima, M.M., Giovanetti, M., de Vasconcelos, J.M., Franco, L., Cardoso, J.F., Vianez-Júnior, J.L.D.S.G., Mir, D., Bello, G., Delatorre, E., Khan, K., Creatore, M., Coelho, G.E., de Oliveira, W.K., Tesh, R., Pybus, O.G., Nunes, M.R.T., Vasconcelos, P.F.C. (2016). Zika virus in the Americas: Early epidemiological and genetic findings. Science, 352, 345-349.

Fauci, A.S., Morens, D.M. (2016). Zika virus in the AmericasYet another Arbovirus threat. N Engl J Med, 374, 601-604.

Ferreira-de-Brito, A., Ribeiro, I.P., Miranda, R.M., Fernandes, R.S., Campos, S.S., Silva, K.A., Castro, M.G., Bonaldo, M. C., Brasil, P., Lourenço-de-Oliveira, R. (2016). First detection of natural infection of Aedes aegypti with Zika virus in Brazil and throughout South America. Mem Inst Oswaldo Cruz, 111, 655-658.

Fontenille, D., Toto, J.C. (2001). Aedes (Stegomyia) albopictus (Skuse), a potential new dengue vector in southern Cameroon. Emerg Infect Dis, 7, 1066-1067.

Forattini, O.P. (1986). Identification of Aedes (Stegomyia) albopictus (Skuse) in Brazil. Rev Saude Publica, 20, 244-245.

Gjenero-Margan, I., Aleraj, B., Krajcar, D., Lesnikar, V., Klobucar, A., Pem-Novosel, I., Kurečić-Filipović, S., Komparak, S., Martić, R., Duričić, S., Betica-Radić, L., Okmadžić, J., Vilibić-Čavlek, T., Babić-Erceg, A., Turković, B., AvsićZupanc, T., Radić, I., Ljubić, M., Sarac, K., Benić, N., Mlinarić-Galinović, G. (2011). Autochthonous dengue fever in Croatia, August-September 2010. Euro Surveill, 16(9), pii: 19805.

Gratz, N.G. (2004). Critical review of the vector status of Aedes albopictus. Med Vet Entomol, 18, 215-227.

Gubler, D.J. (2011). Dengue, Urbanization and Globalization: The Unholy Trinity of the 21(st) Century. Trop Med Health, 39(4 Suppl), 3-11.

Guimera, R., Mossa, S., Turtschi, A., Amaral, L.A. (2005). The worldwide air transportation network: Anomalous centrality, community structure, and cities' global roles. Proc Natl Acad Sci USA, 102, 7794-7799. 
Hanson, S.M., Craig, G.B., Jr. (1994). Cold acclimation, diapause, and geographic origin affect cold hardiness in eggs of Aedes albopictus (Diptera: Culicidae). J Med Entomol, 31, 192-201.

Hawley, W.A., Reiter, P., Copeland, R.S., Pumpuni, C.B., Craig, G.B., Jr. (1987). Aedes albopictus in North America: probable introduction in used tires from northern Asia. Science, 236, 1114-1116.

Jones, K.E., Patel, N.G., Levy, M.A., Storeygard, A., Balk, D., Gittleman, J.L., Daszak, P. (2008). Global trends in emerging infectious diseases. Nature, 451, 990-993.

Jupille, H., Seixas, G., Mousson, L., Sousa, C.A., Failloux, A.B. (2016). Zika virus, a new threat for Europe? PLoS Negl Trop Dis, 10, e0004901.

Kraemer, M.U., Sinka, M.E., Duda, K.A., Mylne, A.Q., Shearer, F.M., Barker, C.M., Lourenco-de-Oliveira, R., Failloux, A.B. (2017). Lessons learned on Zika virus vectors. PLoS Negl Trop Dis, 11, e0005511.

Kramer, L.D., Ebel, G.D. (2003). Dynamics of flavivirus infection in mosquitoes. Adv Virus Res, 60, 187-232.

La Ruche, G., Souarès, Y., Armengaud, A., Peloux-Petiot, F., Delaunay, P., Desprès, P., Lenglet, A., Jourdain, F., LeparcGoffart, I., Charlet, F., Ollier, L., Mantey, K., Mollet, T., Fournier, J.P., Torrents, R., Leitmeyer, K., Hilairet, P., Zeller, H., Van Bortel, W., Dejour-Salamanca, D., Grandadam, M., Gastellu-Etchegorry, M. (2010). First two autochthonous dengue virus infections in metropolitan France, September 2010. Euro Surveill, 15, 19676.

Lourenço-de-Oliveira, R., Failloux, A.B. (2017). High risk for chikungunya virus to initiate an enzootic sylvatic cycle in the tropical Americas. PLoS Negl Trop Dis, 11, e0005698.

Medlock, J.M., Hansford, K.M., Schaffner, F., Versteirt, V., Hendrickx, G., Zeller, H., Van Bortel, W. (2012). A review of the invasive mosquitoes in Europe: ecology, public health risks, and control options. Vector Borne Zoonotic Dis, 12, 435- 447 .

Musso, D., Nilles, E.J., Cao-Lormeau, V.M. (2014). Rapid spread of emerging Zika virus in the Pacific area. Clin Microbiol Infect, 20, O595-O596.

Paupy, C., Delatte, H., Bagny, L., Corbel, V., Fontenille, D. (2009). Aedes albopictus, an arbovirus vector: from the darkness to the light. Microbes Infect, 11, 1177-1185.

Perrings, C., Dehnen-Schmutz, K., Touza, J., Williamson, M. (2005). How to manage biological invasions under globalization. Trends Ecol Evol, 20, 212-215.

Powell, J.R., Gloria-Soria, A., Kotsakiozi, P. (2018). Recent history of Aedes aegypti: Vector genomics and epidemiology records. Bioscience, 68, 854-860.

Raharimalala, F.N., Ravaomanarivo, L.H., Ravelonandro, P., Rafarasoa, L.S., Zouache, K., Tran-Van, V., Mousson, L, Failloux, AB, Hellard, E, Moro, CV, Ralisoa, BO, Mavingui, P. (2012). Biogeography of the two major arbovirus mosquito vectors, Aedes aegypti and Aedes albopictus (Diptera, Culicidae), in Madagascar. Parasit Vectors, 5, 56.

Rasmussen, S.A., Jamieson, D.J., Honein, M.A., Petersen, L.R. (2016). Zika virus and birth defects - Reviewing the evidence for causality. N Engl J Med, 374, 1981-1987.

Rezende, G.L., Martins, A.J., Gentile, C., Farnesi, L.C., PelajoMachado, M., Peixoto, A.A, Valle, D. (2008). Embryonic desiccation resistance in Aedes aegypti: presumptive role of the chitinized serosal cuticle. BMC Dev Biol, 8, 82.
Rohani, A., Wong, Y.C., Zamre, I., Lee, H.L., Zurainee, M.N. (2009). The effect of extrinsic incubation temperature on development of dengue serotype 2 and 4 viruses in Aedes aegypti (L.). Southeast Asian J Trop Med Public Health, 40, 942-950.

Roundy, C.M., Azar, S.R., Brault, A.C., Ebel, G.D., Failloux, A. B., Fernandez-Salas, I., Kitron, U., Kramer, L.D., Lourençode-Oliveira, R., Osorio, J.E., Paploski, I.D., VazquezProkopec, G.M., Ribeiro, G.S., Ritchie, S.A., Tauro, L.B., Vasilakis, N., Weaver, S.C. (2017). Lack of evidence for Zika virus transmission by Culex mosquitoes. Emerg Microbes Infect, 6, e90.

Ruckert, C., Ebel, G.D. (2018). How do virus-mosquito interactions lead to viral emergence? Trends Parasitol, 34, 310-321.

Savage, H.M., Ezike, V.I., Nwankwo, A.C., Spiegel, R., Miller, BR. (1992). First record of breeding populations of Aedes albopictus in continental Africa: implications for arboviral transmission. J Am Mosq Control Assoc, 8, 101-103.

Schaffner, F., Karch, S. (2000). [First report of Aedes albopictus (Skuse, 1984) in metropolitan France]. CR Acad Sci III, 323, 373-375.

Schuffenecker, I., Iteman, I., Michault, A., Murri, S., Frangeul, L., Vaney, M.C., Lavenir, R., Pardigon, N., Reynes, J.M., Pettinelli, F., Biscornet, L., Diancourt, L., Michel, S., Duquerroy, S., Guigon, G., Frenkiel, M.P., Bréhin, A.C., Cubito, N., Desprès, P., Kunst, F., Rey, F.A., Zeller, H., Brisse, S. (2006). Genome microevolution of chikungunya viruses causing the Indian Ocean outbreak. PLoS Med, 3, e 263.

Tatem, A.J., Rogers, D.J., Hay, S.I. (2006). Global transport networks and infectious disease spread. Adv Parasitol, 62, 293-343.

Ten Bosch, Q.A., Clapham, H.E., Lambrechts, L., Duong, V., Buchy, P., Althouse, B.M., Lloyd, A.L., Waller, L.A., Morrison, A.C., Kitron, U., Vazquez-Prokopec, G.M., Scott, T.W., Perkins, T.A. (2018). Contributions from the silent majority dominate dengue virus transmission. PLoS Pathog, 14, e1006965.

Tomasello, D., Schlagenhauf, P. (2013). Chikungunya and dengue autochthonous cases in Europe, 2007-2012. Travel Med Infect Dis, 11, 274-284.

Toto, J.C, Abaga, S., Carnevale, P., Simard, F. (2003). First report of the oriental mosquito Aedes albopictus on the West African island of Bioko, Equatorial Guinea. Med Vet Entomol, 17, 343-346.

Tsetsarkin, K.A., Vanlandingham, D.L., McGee, C.E., Higgs, S. (2007). A single mutation in chikungunya virus affects vector specificity and epidemic potential. PLoS Pathog, 3, e201.

Upham, P., Thomas, C., Gillingwater, D., Raper, D. (2003). Environmental capacity and airport operations: current issues and future prospects. J Air Transp Manag, 9, 145-151.

Vasilakis, N, Weaver, S.C. (2008). The history and evolution of human dengue emergence. Adv Virus Res, 72, 1-76.

Vazeille, M., Moutailler, S., Coudrier, D., Rousseaux, C., Khun, H., Huerre, M., Thiria, J., Dehecq, J.S., Fontenille, D., Schuffenecker, I, Despres, P., Failloux, A.B. (2007). Two Chikungunya isolates from the outbreak of La Réunion (Indian Ocean) exhibit different patterns of infection in the mosquito, Aedes albopictus. PLoS One, 2, e1168.

Venturi, G., Di Luca, M., Fortuna, C., Remoli, M.E., Riccardo, F., Severini, F., Toma, L., Del Manso, M., Benedetti, E., Caporali, M.G., Amendola, A., Fiorentini, C., De Liberato, 
C., Giammattei, R., Romi, R., Pezzotti, P., Rezza, G., Rizzo, C. (2017). Detection of a chikungunya outbreak in Central Italy, August to September 2017. Euro Surveill, 22(39).

Woolhouse, M.E., Gowtage-Sequeria, S. (2005). Host range and emerging and reemerging pathogens. Emerg Infect Dis, 11, 1842-1847.

Xiao, F.Z., Zhang, Y., Deng, Y.Q., He, S., Xie, H.G., Zhou, X. N., Yan, Y.S. (2014). The effect of temperature on the extrinsicincubation period and infection rate of dengue virus serotype 2 infection in Aedes albopictus. Arch Virol, 159, 3053-3057.

Yactayo, S., Staples, J.E., Millot, V., Cibrelus, L., RamonPardo, P. (2016). Epidemiology of Chikungunya in the Americas. J Infect Dis, 214, S441-S445.

Zachcial, M., Heideloff, C. (2003). ISL Shipping Statistics Yearbook 2003. Logistics BIoSEa, editor.

Citation de l'article : Failloux, A.-B. (2018). Les moustiques vecteurs d'arbovirus : une histoire sans fin. Biologie Aujourd'hui, 212, 89-99 